\title{
Patellar Giant-Cell Tumor: a Case Report
}

\author{
Istan Irmansyah Irsan ${ }^{1,2}$, Satria Pandu Persada Isma ${ }^{1,2}$, Rakhmad Aditya Hernawan ${ }^{1,2^{*}}$ \\ ${ }^{1}$ Department of Orthopaedic and Traumatology, Faculty of Medicine, University of Brawijaya, Malang, Indonesia \\ ${ }^{2}$ Saiful Anwar Hospital, Malang, Indonesia
}

\begin{abstract}
Giant cell tumor (GCT) found mostly in the long bones metaphysis or epiphysis. GCT usually occur between the ages of a third and fourth decade and locally aggressive. Giant-Cell Tumor seldom affects the patella. Multicentric forms rarely reported. A fourteen-year-old female with a lump at her left patella since one-year associated with slight pain that aggravated by activity for six months. Plain $X$-ray left knee $A P$, and lateral views reveal expansile lytic lesion in left patella with thinning of the anteroinferior cortex and sclerotic septa within. MRI of left knee shows approximately $3 \times 2 \times 2$ $\mathrm{cm}$ heterogeneous lobulated expansile soft tissue mass in left patella extending up to the patellofemoral joint with fluid-fluid appearance. From fine needle aspiration cytology, resulting giant-cell tumor with the differential diagnosis of an aneurysmal bone cyst. The operation already performed. Curettage, bone graft, and a biopsy taken. An immunocytochemical smear was performed and confirmed as a patellar giant-cell tumor. Six months after excision of the tumor, the patient complained no arthralgia and have a full range of motion for the knee.
\end{abstract}

Keywords: Giant-cell tumor, knee lump, knee pain, patella.

\section{INTRODUCTION}

Many cases of giant cell tumor (GCT) found in long bones metaphysis or epiphysis. GCT usually occur between the ages of a third and fourth decade, it is locally aggressive but also has metastatic potential $[1,2]$. This lesion most often located around the knee joint, originates at the epiphysis, spreads to the metaphysis and makes the cortex erode in $25 \%$ of the cases. Ten percent of these tumors have a malignant course [3]. The reported incidence of patellar GCT was less than one percent [4]. This article reported a left patellar GCT which diagnosed on fine needle aspiration cytology, magnetic resonance imaging and already performed excision, curettage, and bone cement.

\section{CASE REPORT}

A fourteen-year-old female at Malang, East Java, presented with a lump since one-year that grows progressively at her left patella associated with slight pain that has aggravated with activity for six months. It measured $42 \mathrm{~cm}$ in its diameters with a normal skin surface. There are no pulmonary and other systemic symptoms. The other laboratory result was within limits.

The patient underwent plain left anteroposterior (AP) and lateral views knee $x$-ray. Radiograph reveal expansive lytic lesion in left

\footnotetext{
* Correspondence address: Rakhmad Aditya Hernawan

Email : aditflea@gmail.com

Address: Dept. Orthopaedic \& Traumatology, Faculty of Medicine, University of Brawijaya, Veteran Malang, Malang 65145.
}

patella with sclerotic septa within and thinning of the anteroinferior cortex (Fig. 1).

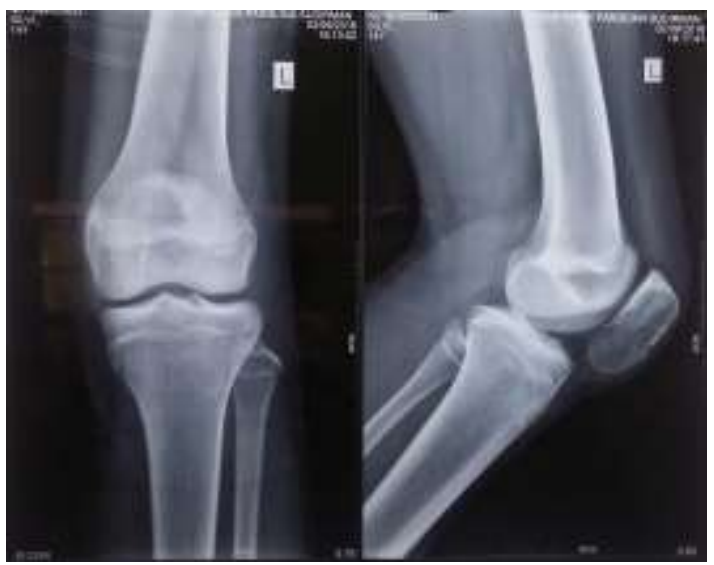

Figure 1. The lateral and anteroposterior plain x-ray shows expansive lytic lesion in the left patella

The patient underwent MRI of the left knee. $T_{1}$ and $T_{2}$ weighted images obtained in sagittal and axial planes (Fig. 2 and 3). MRI of left knee shows approximately $3 \times 2 \times 2 \mathrm{~cm}$ heterogeneous lobulated expansive soft tissue lump in left patella extending up to the articular surface, and a fluid-fluid level observed. The possible differential diagnosis from MRI includes the giant-cell tumor or an aneurysmal bone cyst. Luckily, the articular cartilage at the posterosuperior part is still intact.

For diagnosis confirmation, fine needle aspiration performed at the patellar anteroinferior portion. Histopathological finding still cannot differentiate whether this is the giant-cell tumor or an aneurysmal bone cyst although 
multi-nucleated giant cell was already found (Fig. 4).

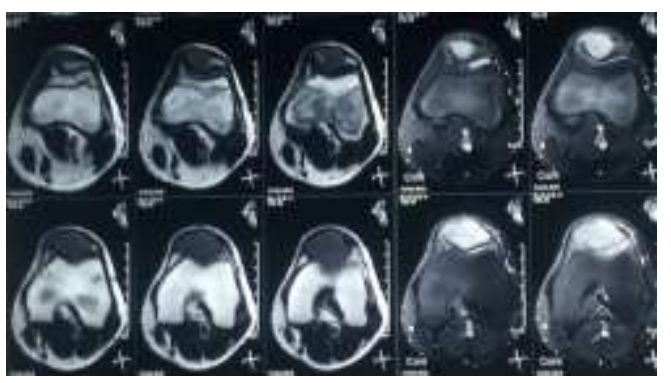

Figure 2. $T_{1}$ and $T_{2}$ axial MRI show defect in an anteroinferior aspect of the patella

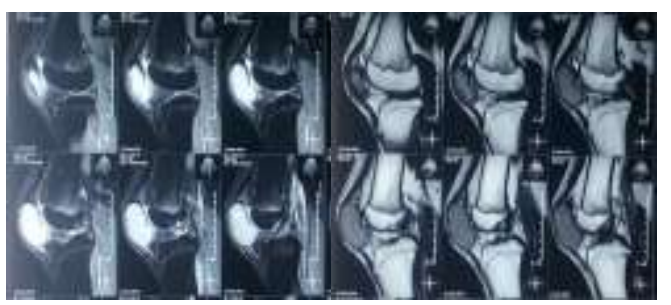

Figure 3. Sagittal $T_{1}$ and $T_{2} M R I$ show a fluid-fluid level
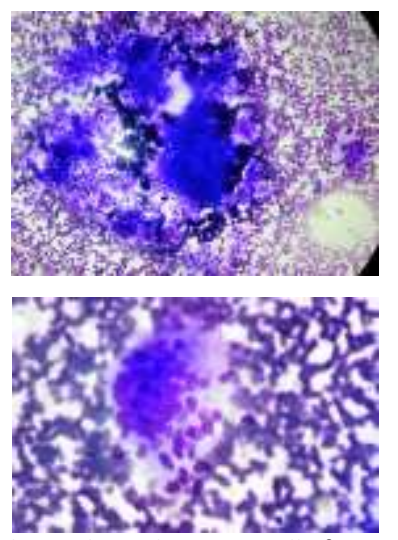

Figure 4. Multi-nucleated giant cell from fine needle aspiration

We performed lesion curettage and bone substitution with general anesthesia. These procedures performed through the longitudinal incision at approximately $10 \mathrm{~cm}$ above the area. The incision was made with an oval shape, the periosteum was detached with a raspatory from the patellar surface. From appearance, the tumor was solid, yellow-brown coloring with small blood retention (Fig. 5). The tumor macroscopically looks like a GCT. We curetted the contents carefully and filled approximately 5 $\mathrm{g}$ of calcium hydroxy-apatite ceramic to the defect and fixed it with two screws. Plain radiograph of the left knee anteroposterior (AP) and lateral views post-surgery taken to confirm the bone substitution component fills in the defect (Fig. 6).

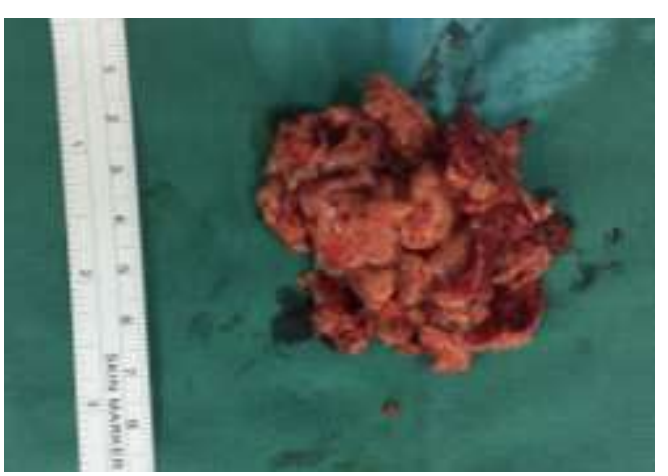

Figure 5. Macroscopically of the tumors, solid and yellow-brown coloring with small blood retention

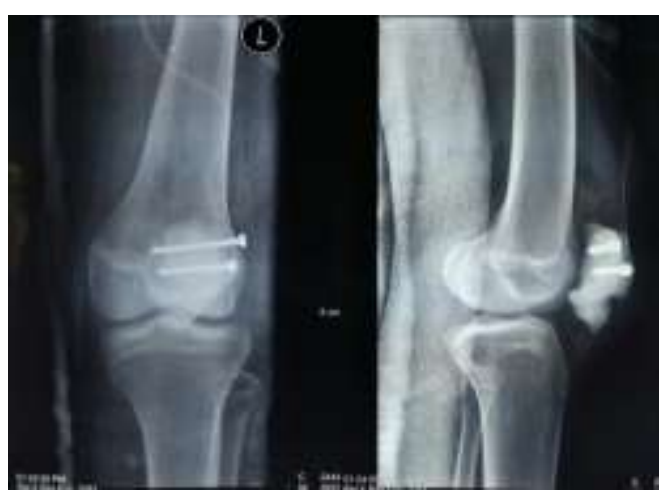

Figure 6. Post surgery AP and lateral left knee plain x-ray

Histopathological examination was performed after the surgery and showed multinucleated giant cell with more than 20 nuclei, spindle-shaped short cells, calcification and bone tissues with few mitotic figures and no cellular atypia observed. By this findings, the lesion diagnosed as a GCT instead of an aneurysmal bone cyst (Fig. 7). Immunohistochemical was performed to confirm the diagnosis and found a positive result in CD68 staining (Fig. 8). The left patellar giant-cell tumor confirmed.

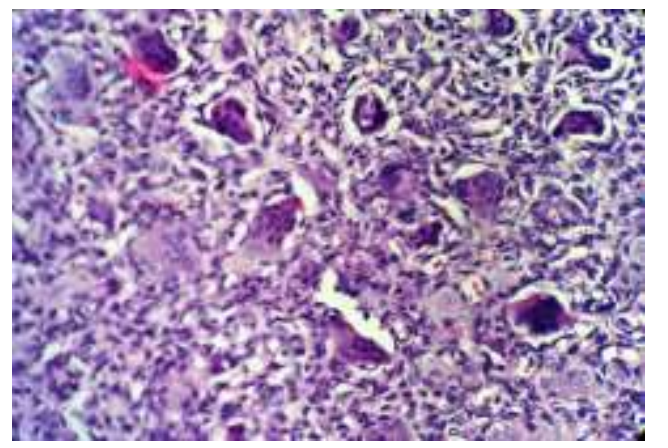

Figure 7. Histopathological examination shows multinucleated giant cell with more than 20 nuclei, spindle-shaped short cells, calcification and bone tissues 


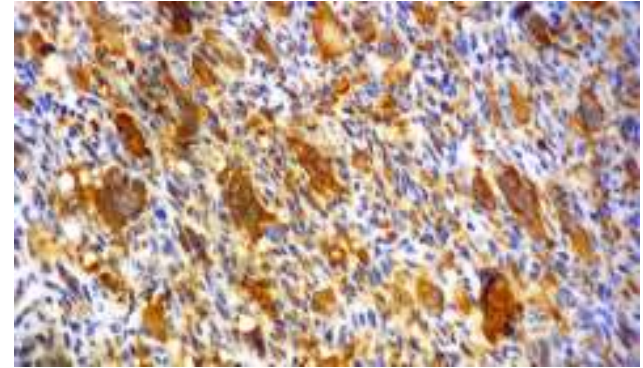

Figure 8. The immunohistochemical show positive result of CD68 staining

The knee was then protected with a splint for two weeks after the operation. One week after the surgery the suture was removed and then the patient begins to a range of motion and weightbearing exercise. Full weight-bearing allowed four weeks after the surgery. The patient regains a full motion six months after surgery and has no arthralgia of the knee.

\section{DISCUSSION}

Bone tumors seldom affected the patella. A survey held by the Japanese Orthopedic Association, 403 primary bone tumor cases were reported from 1972 to 2003 . Most of these cases, $70.5 \%$ involving the lower extremities [1]. However, tumors affected the patella were only $0.5 \%$ (75) of all cases. Of these, 71 were benign bone tumors, that is 22 was diagnosed with GCT, 14 was solitary exostosis, and 24 was chondroblastoma. The patellar GCT reported in the international literature was only less than 50 cases worldwide [2].

Clinically, the patients with patellar GCT usually complained about lump and pain at the knee [3-7]. From the inspection, we found lump, redness, swelling, and effusion. From the palpation, we found local heat, tenderness, and crepitus. An attempt to move the affected part shows the decrease of motion range [3-7]. The laboratory results in severe cases showed a serum alkaline phosphatase (ALP) increasing and also erythrocyte sedimentation rate (ESR) [8].

Radiographs showed an osteolytic lesion, destruction of the bone, and sometimes, soap bubble appearance [3]. Magnetic resonance imaging (MRI) showed expansive soft tissue mass of the patella, and also some evidence of adjacent tissues and sclerotin [8]. On GCT patient, the chest $x$-ray is necessary to determine a possibility of lung metastasis and also bone scintigraphy for metastasis on other bones.

A fine needle or incisional biopsy are recommended to establish the diagnosis. Microscopically, GCT featuring multinucleated giant cells, a few mitotic spindle-shaped short cells, and bone tissue calcification should be distinguished from a chondroblastoma or an aneurysmal bone cyst. An aggressive GCT histologically showed a nuclear atypia with compact stroma, hyperchromatic, frequent mitotic figures, and the unevenly distributed giant cells [7].

Curettage that combines with cryosurgery may be the choice in the early stage of patellar GCT with the risk of recurrence about 35\% [10]. For Enneking stage $2 \mathrm{GCT}$, the therapy should be partial patellectomy or curettage intralesional with bone substitution and extensor mechanism reconstruction is the first choice in the management of patellar GCT. But for Enneking stage 2 to $3 \mathrm{GCT}$, patellectomy combined with adjuvant treatment for metastasis is recommended [8]. In the advanced cases, only treatment left that offers best functional results is resection [2]. Novel treatment includes using osteoarticular grafts for reconstruction after patellar resection in advanced cases may be a feasible option [10].

\section{CONCLUSION}

Patellar GCT can be mistaken for other tumors of soft tissues and bone. Fine needle or biopsy would be diagnostic if an adequate specimen is obtained and canal so confirms with immunohistochemical staining. In the early stage, curettage accompanied with bone substitution and cryosurgery may be attempted, and patellectomy for the late stage. For future reconstruction procedure, osteoarticular grafts can be used after resection that may be a feasible option for the advanced cases.

\section{REFERENCES}

[1] Singh, V.P., N. Kannan, H.G. Mukhopadhyay, S. Mukherjee, R. Lakhtakia. 1996. Giant cell tumor of bone: an unusual metastatic pattern. Med. J. Armed Forces India. 52(4). 260-262

[2] Paudel, S., P. Kayastha, P. Pokhrel, A. Shah, R.K. Ghimire, M.A. Ansari. 2012. Giant cell tumor of patella. J. Inst. Med. 34. 58-60.

[3] Hutagalung, E.U., S. Gumay, B. Budiatmoka 2005. Neoplasma tulang: diagnosis dan terapi. Galaxy Puspa Mega. Bekasi. 41-43.

[4] Yoshida, Y., T. Kojima, M. Taniguchi, S. Osaka, Y. Tokuhashi. 2012. Giant-cell tumor of the patella. Acta Med. Okayama. 66. 7376.

[5] Ofluoglu, O., R. Donthineni. 2007. latrogenic seeding of a giant cell tumor of 
the patella to the proximal tibia. Clin. Orthop. Relat. Res. 465. 260-264.

[6] Malhotra, R., L. Sharma, V. Kumar, A.R. Nataraj. 2010. Giant cell tumor of the patella and its management using a patella, patellar tendon, and tibial tubercle allograft. Knee Surg. Sports Traumatol. Arthrosc. 18. 167-169.

[7] Chakravarty, U., G. Chakraverty. 2009. Late presentation of GCT of the patella. Int. J. Orthop. Surg. 12-13.

[8] Song, M., Z. Zhang, Y. Wu, K. Ma, M. Lu. 2015. Primary tumors of the patella. World J. Surg. Oncol. 13. 163.

[9] Christopher, D. 2002. Pathology and genetics of tumours of soft tissue and bone. International Agency for Research on Cancer. France. 309-312.

[10] Carnevale, P.G. 1987. Sometimes malignant tumors of bone, in Campbell's operative orthopedics, $7^{\text {th }}$ Ed. St. Louis. Mosby. 765805. 